\title{
agriculture
}

ISSN 2073-4395

www.mdpi.com/journal/agriculture

Article

\section{Silencing of $M g$-pat-10 and $M g$-unc-87 in the Plant Parasitic Nematode Meloidogyne graminicola Using siRNAs}

\author{
Joseph Nsengimana ${ }^{\dagger}$, Lander Bauters, Annelies Haegeman and Godelieve Gheysen * \\ Department of Molecular Biotechnology, Ghent University, 653 Coupure links, 9000 Gent, Belgium; \\ E-Mails: lander.bauters@ugent.be (L.B.); annelies.haegeman@ugent.be (A.H.)
}

$\dagger$ Current Address: Department of Applied Biology, Kigali Institute of Science and Technology (KIST), 3900 Avenue de la Paix, 250 Kigali, Rwanda; E-Mail: josephnsengimana@yahoo.fr

* Author to whom correspondence should be addressed; E-Mail: godelieve.gheysen@ugent.be; Tel.: +32-9-264-59-68; Fax: +32-9-264-62-19.

Received: 27 April 2013; in revised form: 6 August 2013 / Accepted: 22 August 2013 /

Published: 18 September 2013

\begin{abstract}
Until recently, the standard method for RNA interference (RNAi)-based reverse genetics in plant parasitic nematodes (PPNs) was based on the use of long double-stranded RNA (dsRNA). This increased the chance of off-target gene silencing through interactions between different short interfering RNAs (siRNAs) and non-cognate mRNA targets. In this work, we applied gene-specific knockdown of $M g$-pat-10 and $M g$-unc-87 of the root knot nematode Meloidogyne graminicola, using discrete 21 bp siRNAs. The homologue of Mg-pat-10 in C. elegans encodes body wall troponin C, which is essential for muscle contraction, whereas the homologue of $M g-u n c-87$ encodes two proteins involved in maintenance of the structure of myofilaments in the body wall muscle of $C$. elegans. The knockdown at the transcript level, as seen by semi-quantitative RT-PCR analysis, indicates that the $M g$-pat-10 gene was silenced after soaking the nematodes in a specific siRNA for $48 \mathrm{~h}$. At $72 \mathrm{~h}$ post-soaking, the $M g$-pat-10 mRNA level was similar to the control, indicating the recovery of expression between $48 \mathrm{~h}$ and $72 \mathrm{~h}$ post-soaking. For $M g-u n c-87$ the nematodes started to recover from siRNA silencing $24 \mathrm{~h}$ after thorough washing. A migration assay showed that for the nematodes that were soaked in the control (siRNA of $\beta$-1,4-endoglucanase), $77 \%$ of the nematodes completed migration through the column in a $12 \mathrm{~h}$ period. By comparison with the control, nematodes incubated in the siRNA of pat-10 or unc-87 were significantly inhibited in their motility. After $12 \mathrm{~h}$, only $6.3 \%$ of the juveniles incubated in the $M g$-pat-10 siRNA and $9.3 \%$ of those incubated in $M g-u n c-87$
\end{abstract}


siRNA had migrated through the column, representing $91.8 \%$ and $87.9 \%$ inhibition respectively compared to the control. In the present work, we demonstrated that M. graminicola is readily susceptible to siRNAs of two genes involved in nematode motility. This is an important contribution to the progressive use of siRNA for functional analysis. Moreover, the application of RNAi in PPNs opens the way for environmentally friendly control of M. graminicola.

Keywords: short interfering RNA (siRNA); Meloidogyne graminicola (Mg); Mg-pat-10; $M g$-unc-87; migration assay; expression analysis

\section{Introduction}

Plant-parasitic nematodes (PPNs), which include the genus Meloidogyne, are responsible for some of the most damaging biotic stresses on crops [1]. Rice plants infected by the root-knot nematode $M$. graminicola are characterized by a number of distinctive signs; the development of abnormal swellings of the root tip known as galls, as well as yellowing, stunting and wilting of the plants [2]. The current practice operating to control PPNs is through the integrated application of several procedures; nematicides, resistant crop varieties, and biological control strategies such as the use of trap crops, natural enemies and cultural practice [3]. However, these approaches are becoming increasingly unsatisfactory due to the proliferation of PPNs through the continuous and intensive cultivation of susceptible varieties. Significant progress has been made with the use of RNAi in PPNs and this may be advantageous for parasite control through plants engineered to express PPN-specific transcripts. Recently it was demonstrated that RNAi application was a successful approach to functional genomics and nematode control [4]. The RNAi pathway elicits sequence-specific silencing of target mRNA by means of introduction of homologous double stranded RNA [5,6]. For the free living nematode $C$. elegans, dsRNA can be delivered by feeding the nematodes with bacteria producing dsRNA from a plasmid. In contrast to $C$. elegans, PPNs rarely feed outside of their host. Rosso et al. [7] stated that for PPNs, eggs and hatched J2 can be used for soaking. Soaking of J2 of the cyst nematode $(\mathrm{CN})$ Globodera pallida in dsRNA was used to target five flp genes and contrary to C. elegans, in which neuronal tissues are refractory to RNAi, soaking J2 in dsRNA induced a strong depletion in flp transcripts and motility alteration phenotypes [8]. It was noted surprisingly that $f l p$ genes from $G$. pallida seem particularly sensitive to RNAi because dsRNA concentrations as low as $10^{-4}-10^{-9} \mu \mathrm{g} / \mu \mathrm{L}$ were sufficient to trigger silencing. Mi-CRT, a calreticulin gene expressed in the esophageal glands of Meloidogyne incognita can be silenced using siRNAs as shown by Arguel et al. [4], who further demonstrated that this knocking of $M i-C R T$ was not persistent.

In the present work, we evaluated the performance of discrete $21 \mathrm{bp}$ siRNAs, targeting unc-87 and pat-10 in M. graminicola. In C. elegans, pat-10 encodes body wall muscle troponin C, the calcium-binding component of actin filaments. RNAi targeting of this gene in $C$. elegans showed that the knocking down of pat-10 leads to paralysis, embryonic lethality and maternal sterility [9]. In C. elegans, through alternative splicing $u n c-87$ encodes two proteins which are required to maintain the structure of myofilaments in body wall muscle cells. Unc-87 resembles the C-terminal repeat 
region of calponin and associates with thin F-actin filaments [10,11]. An RNAi study using dsRNA to unc-87 in C. elegans by Simmer et al. showed that the knockdown of this gene results in uncoordinated locomotion of the nematode [12].

\section{Materials and Methods}

\subsection{Oligonucleotides Design and siRNA Synthesis}

Homologues of $C$. elegans pat-10 (F54C1.7) and unc-87 (F08B6.4) as well as a homologue of M. incognita $\beta$-1,4-endoglucanase (AAK21881.1) were identified from an EST dataset of M. graminicola by a tblastn search from Haegeman et al. [13]. Using DNA translation tools [14], eligible ORFs were selected to mark the region suited for siRNA target design. The siRNA target finder [15] and Silencer siRNA construction kit (Ambion) were used to design and synthesize siRNAs. The oligonucleotides used for the synthesis were supplied by Biolegio [16] and are shown in Table 1. Four pairs of oligonucleotides, unca, uncb, uncc and uncbM for the unc-87 gene; pata, patb, patc and patbM for the pat-10 gene were constructed. In the same way, the oligonucleotides for the $\beta$-1,4-endoglucanase (Mg-eng) gene were designed. The synthesis of siRNAs was performed according to the protocol provided by Ambion and all the reagents used were provided by the Silencer $^{\circledR}$ siRNA Construction Kit.

Table 1. Oligonucleotides (senses and antisenses) used to synthesize short interfering RNAs (siRNAs).

\begin{tabular}{cc}
\hline SiRNA & Oligonucleotides \\
\hline \multirow{2}{*}{ unca } & Antisense: 5'-AAGAAAAAATCCGTGCTAGTGCCTGTCTC-3' \\
& Sense: 5'-AACACTAGCACGGATTTTTCCCTGTCTC-3' \\
uncb & Antisense: 5'-AAAAAGGAATGGTCAGCTTCGCCTGTCTC-3' \\
& Sense: 5'-AACGAAGCTGACCATTCCTTTCCTGTCTC-3' \\
uncbM & Antisense: 5'-AAAAAGGAATGGTCAGCTTCGCCTGTCTC-3' \\
& Sense: 5'-AAGGAAGCTGACCATTCCTTTCCTGTCTC-3' \\
uncc & Antisense: 5'-AATATCCAGAGGAGGCTGAAACCTGTCTC-3' \\
& Sense: 5'-AATTTCAGCCTCCTCTGGATACCTGTCTC-3' \\
pata & Antisense: 5'-AAATGGCCGAAAATATTGAAGCCTGTCTC-3' \\
& Sense: 5'-AACTTCAATATTTTCGGCCATCCTGTCTC-3' \\
patb & Antisense: 5'-AACCCTTCGAAAATTAATCCGCCTGTCTC -3' \\
patbM & Sense: 5'-AACGATTAATTTTCGAAGGGCCTGTCTC-3' \\
& Antisense: 5'-AAAAAGGAATGGTCAGCTTCGCCTGTCTC-3' \\
patc & Sense:5'-AACCATTAATTTTCGAAGGGCCTGTCTC-3' \\
& Antisense: 5'-AATTAATGGCTGGAGAGACTGCCTGTCTC-3' \\
enga & Sense: 5'-AACAGTCTCTCCAGCCATTAACCTGTCTC-3' \\
\hline
\end{tabular}


Table 1. Cont.

\begin{tabular}{cc}
\hline SiRNA & Oligonucleotides \\
\hline \multirow{2}{*}{ engb } & Antisense: 5'-AATGGGAATGTTGTTCGTGCTCCTGTCTC-3' \\
& Sense: 5'-AAAGCACGAACAACATTCCCACCTGTCTC-3' \\
engbM & Antisense: 5'-AATGGGAATGTTGTTCGTGCTCCTGTCTC-3' \\
& Sense: 5'-AATGCACGAACAACATTCCCACCTGTCTC-3' \\
engc & Antisense: 5'-AACGCTGTTCTTACTCAAGTTCCTGTCTC-3' \\
& Sense: 5'-AAAACTTGAGTAAGAACAGCGCCTGTCTC-3' \\
\hline
\end{tabular}

Table 1, both sense and antisense sequences of oligonucleotides were designed. The T7 promoter sequence (5'-CCTGTCTC-3') was added for the siRNA synthesis.

The fragments for siRNA synthesis were chosen inside the ORF. The fragment "a" was chosen in the beginning of the ORF, " $\mathrm{b}$ " in the middle and "c" at the end of the ORF. A mismatch (M) was designed in the fragment chosen in the middle (b) in an attempt to increase the chance for siRNA success [17].

\subsection{Nematode Culture, Collection and Soaking in siRNA}

The M. graminicola culture was provided by Dirk De Waele (Catholic University of Leuven, Leuven, Belgium) and was originally isolated in the Philippines. It has since been maintained on rice cv Nipponbare and on the grass Echinocloa crus-galli. The culture was kept in a plant room at $28{ }^{\circ} \mathrm{C}$ under a light regime of $12 \mathrm{~h}$ light/12 h darkness, and 70\%-75\% relative humidity. Stage 2 juveniles (J2) were extracted from a three month old culture using the modified Baermann method [18]. Approximately 1000 nematodes were mixed with siRNA $(50 \mathrm{ng} / \mu \mathrm{L})$ resuspended in $50 \mu \mathrm{L}$ of distilled water and this was incubated for $24 \mathrm{~h}$ on a rotator at room temperature.

The nematodes were then washed copiously with tap water to remove external siRNA. Then the characteristic phenotypes were checked using a stereomicroscope at the following time points: immediately after washing and removal of external siRNA $(0 \mathrm{~h})$, at $6 \mathrm{~h}$, at $24 \mathrm{~h}$, at $48 \mathrm{~h}$ and at $72 \mathrm{~h}$. The posture and activity of the nematodes incubated in siRNAs targeting $\beta$-1,4-endoglucanase was used as a control. About 300 nematodes from each treatment were used for expression analysis and another 300 for the migration assay. Three independent replicates for each treatment were performed.

\subsection{Migration Assay and Expression Analysis}

Migration assays were used in order to have a more quantitative measurement of the RNAi-effect on nematode mobility. Approximately 300 treated or control nematodes were added to the top of pre-moistened sand columns made by filling a $5 \mathrm{~cm}$ long tube $(5 \mathrm{~mm}$ internal diameter) with washed, coarse river sand and covering the base of the tube with miracloth. The columns were placed vertically in collection vials containing sufficient water with rice root exudates and this covered the base of the column. To obtain rice root exudates, germinating rice seedlings were incubated for $24 \mathrm{~h}$ in a collection vial full of water.

The nematodes incubated in patb-, uncb- and in the control eng-siRNA were counted by microscope after migration. Four columns were used for each treatment and the number of nematodes migrating through the columns into the collection vials was counted after $12 \mathrm{~h}$. A second nematode collection 
and counting was done after $24 \mathrm{~h}$. One-way ANOVA and Tukey's HSD test using the SPSS 16.0 package were used to analyze the results. For gene expression analysis, approximately 300 nematodes were thoroughly washed with sterile water and mRNAs were extracted using a sonication and trizol based protocol (Invitrogen Corporation 2003, Merlbeke, Belgium). The extracted RNA was treated with DNAse I (Invitrogen, Merlbeke, Belgium) and was used as a template for cDNA synthesis using SuperScript $^{\mathrm{TM}}$ II RNase H-Reverse Transcriptase, with an oligo (dT) primer and $10 \mathrm{mM}$ dNTP mix (Invitrogen, Merlbeke, Belgium). The cDNA obtained was used for semi-quantitative PCR.

The following program was used: $2 \mathrm{~min}$ at $95{ }^{\circ} \mathrm{C}$, followed by 30 cycles of $\left(30 \mathrm{~s}\right.$ at $95{ }^{\circ} \mathrm{C}, 45 \mathrm{~s}$ at $58{ }^{\circ} \mathrm{C}$ and $30 \mathrm{~s}$ at $72{ }^{\circ} \mathrm{C}$ ), 2 min at $72{ }^{\circ} \mathrm{C}$. At 30 cycles, $10 \mu \mathrm{L}$ of the PCR reaction mix was withdrawn from the PCR tubes and the reaction proceeded for an additional three cycles. Due to the small amount of material (300 nematodes), 33 cycles were needed to get a good signal. The PCR products were loaded on a $1.5 \%$ agarose gel in $0.5 \times$ TAE buffer and electrophoresis was applied for 20 min at $100 \mathrm{~V}$. The different bands were visualized with ethidium bromide under UV light.

For expression analysis, RT-PCR was carried out. Tubulin (Mg-Contig 16812-71), a house-keeping gene whose amplification is easy to perform was used to normalize the amounts of different cDNA templates, while nematodes soaked in $\beta-1,4$-endoglucanase siRNA as well as in water (data not shown for water) were used as a negative control for general siRNA toxicity and for checking the specificity of gene silencing. Gene-specific forward and reverse primers to amplify a 291-bp fragment of pat-10, a 380-bp fragment of unc-87, a 158-bp fragment of tubulin were used (Table 2).

Table 2. Primers used for RT-PCR of Mg-pat-10, Mg-unc-87, Mg-eng and Tubulin.

\begin{tabular}{ccc}
\hline Gene & Fragment Length & Primer Name and Sequence \\
\hline \multirow{2}{*}{ Mg-pat-10 } & $291-\mathrm{bp}$ & $M g$-pat-F 5'-CAACGTTTCCTCTCTTAATTTTTC-3' \\
& & $M g$-pat-R 5'-TTCGAAGGGTTTTCTCATCAA-3' \\
Mg-unc-87 & 380 -bp & $M g$-unc-F 5'-GATTTGGAGCCTCTTCCAGA-3' \\
& & $M g$-unc-R 5'-TATTCCGGATGGGCAGTATC-3' \\
Mg-tubulin & 158 -bp & $M g$-Tub-F 5'-TCTGGCATAAATAAAATAAGCGAGT-3' \\
& & $M g$-Tub-R 5'-TCAAGATGCAACTGTTGAGGA-3' \\
Mg-eng & 160 -bp & $M g$-eng F 5'-TAGCAGCTAACCCGCCTTATG-3' \\
& & $M g$-eng R 5'-TAGTGCCTCAGGGAAATTGC-3' \\
\hline
\end{tabular}

\section{Results}

\subsection{Mg-pat-10 and Mg-unc-87 Genes Can Be Silenced Using siRNA}

The two target genes for $M$. graminicola were selected because they have similarity with their homologues in C. elegans: $M g$-pat-10 is $91 \%$ similar, $\left(E_{\text {value }}=2^{-23}\right.$ ) while $M g$-unc- 87 is $88 \%$ $\operatorname{similar}\left(E_{\text {value }}=2^{-8}\right)[19]$.

Moreover, the silencing of pat-10 and unc-87 in C. elegans elicits an immediate visible characteristic paralysis of the model nematode. Previous reports on in vitro RNAi in the cyst nematode G. pallida and the root-knot nematode $M$. incognita have shown that high concentrations of non-specific dsRNA induced profound phenotypic changes in the infective juveniles of the nematodes $[8,20]$. With this in mind, we wanted to confirm that a disrupted motility was not due to any general toxic or inhibitory action of the siRNA rather than to the specific silencing of the genes. 
Therefore, siRNAs designed against $\beta-1,4$-endoglucanase sequence of $M$. graminicola were used as a control during the investigation on the effect of siRNA of $M g$-pat-10 and $M g$-unc-87 in M. graminicola. It was demonstrated that $\beta-1,4-$-endoglucanases, secreted through the stylet of PPN, play a role in the softening of cell walls during penetration of the root epidermis and migration of the juveniles [21], and silencing of this gene is not expected to affect motility. An initial screening was done to identify the most efficient siRNAs for each gene (Table 1) by incubating the nematodes in each siRNA. Based on the RT-PCR results and observations of the phenotypes of the incubated nematodes using stereomicroscopy (data not shown), we found that the nematodes soaked in siRNAs designed in the middle (patb and uncb) of the genes were good candidates for thorough investigation. For other siRNAs (pata, patc, unca, uncc), no difference in expression level was observed. While nematodes soaked in Mg-eng-siRNA (or water) showed normal sinusoidal movement, silencing of Mg-pat-10 resulted in profound inhibition of motility. Some of the nematodes incubated in siRNA of Mg-pat-10 were paralyzed and rigid with a hooked head; others barely moved. The nematodes incubated in siRNA of $M g$-unc- 87 were coiled with less movement. Similar results were obtained in all three replicates.

The results of the expression analysis (RT-PCR) after incubation of the nematodes in siRNAs mentioned above are presented in Figure 1. Figure 2 shows the results of the nematode migration assay.

Figure 1. Expression analysis of $M g$-pat-10 and $u n c-87$ after siRNA-treatment. The first panel (a) shows the expression analysis of $M g$-pat-10, the second panel (b) $M g$-unc-87 (the middle lane that is not labeled contains a ladder) and the third panel (c), Mg-tubulin mRNA levels following the treatment by siRNA of $M g$-pat-10 or Mg-unc-87. Lanes 1-5, panel (a) and (b): Expression status after treatment by siRNAs for target genes at the following time points: Lane 1, $0 \mathrm{~h}$ after treatment; lane 2, $6 \mathrm{~h}$; lane 3, $24 \mathrm{~h}$; lane 4, $48 \mathrm{~h}$; lane 5, $72 \mathrm{~h}$. For each panel lane 6 illustrates the control expression of the gene after Mg-eng siRNA treatment.

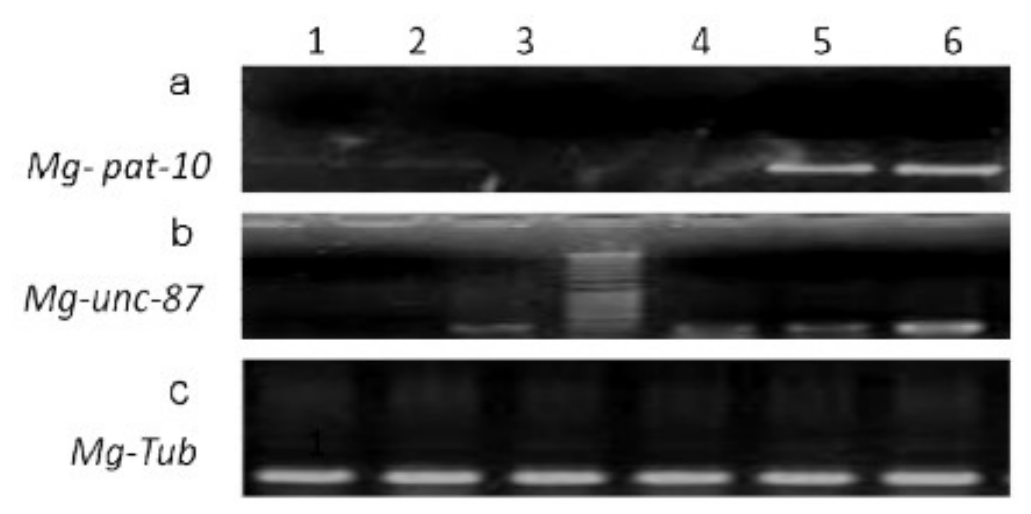

As can be seen in Figure 1 on panel (a), Mg-pat-10 was silenced from 0-48 h after incubation with pat-siRNA. At $72 \mathrm{~h}$ (lane 5) the $M g$-pat-10 mRNA level was similar to the control (lane 6). This means that the recovery of $M g$-pat-10 expression occurred between $48 \mathrm{~h}$ and $72 \mathrm{~h}$ after washing. For $M g$-unc- 87 in panel (b) partial recovery of mRNA was already visible after $24 \mathrm{~h}$ (lane 3), but this was still not complete at $72 \mathrm{~h}$ (lane 5). Lane 6 shows gene expression immediately after soaking in siRNA of Mg-eng demonstrating that expression of $M g$-pat-10 (a) or $M g$-unc-87 (b) was not affected by 
non-specific siRNA effects. With the panel (c) it was observed that expression of the house-keeping gene tubulin was not affected by siRNA treatment against $M g$-pat-10, Mg-unc-87.

The siRNA synthesized using oligonucleotides with a mismatch did not produce any observable aberrant phenotypes. In addition, no difference was noticed in the expression of the target gene after incubation in siRNAs with a mismatch or siRNAs to the control gene (data not shown).

\subsection{Silencing of Mg-pat-10 and Mg-unc-87 Impairs Migration Capacity of $\mathrm{M}$. graminicola}

To quantify the reduction in motility after silencing of $M g$-pat-10 and Mg-unc-87, M. graminicola juveniles were assessed for impaired motility in a sand column migration assay. Under normal conditions, nematodes migrate downward through the sand column. In contrast, nematodes with disrupted mobility have an impaired ability to complete this migration. For nematodes that were incubated for $24 \mathrm{~h}$ in siRNA of $\beta$-1,4-endoglucanase (negative control), a mean migration of $77 \% \pm 3.15 \%(n=4)$ was recorded in a $12 \mathrm{~h}$ period. In contrast, nematodes incubated in siRNA of Mg-pat-10 and Mg-unc-87 showed significant inhibition of motility $(n=4, p=0.01)$. After $12 \mathrm{~h}$, only $6.3 \% \pm 1.19 \%$ of the juveniles incubated in $M g$-pat-10 siRNA and $9.3 \pm 1.21$ of those incubated in Mg-unc-87 siRNA had migrated through the column representing a $91.8 \%$ and $87.9 \%$ inhibition of the control-soaked nematodes, respectively (Figure 2). However, after $24 \mathrm{~h}$ the number of nematodes that completed the migration through the sand column was high in all conditions and the difference between the siRNA treated nematodes and the control was not significant anymore in terms of their migration through the sand column.

Figure 2. Nematode migration assay. Migration assay results at two time points; 12 and $24 \mathrm{~h}$. Three bars show the \% of nematodes that migrated through the sand column at the given time points and read as follows from left to right: Black bar, nematodes incubated in siRNA of control Mg-eng; White, nematodes incubated in siRNA of Mg-pat-10; Gray, nematodes incubated in siRNA of $M g-u n c-87 . *$ indicates significant difference between the treatment and the control.

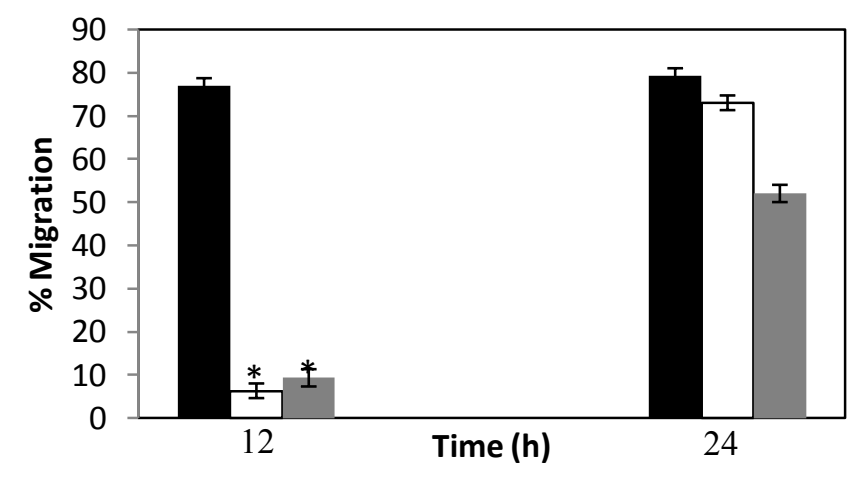




\section{Discussion}

In this work, the effect of small interfering RNA (siRNA) on functional gene knock-down was analyzed in M. graminicola. Two genes, $M g$-pat-10 and $M g$-unc- 87 encoding body wall muscle proteins in nematodes were chosen as the siRNA targets in M. graminicola. The RT-PCR analysis of mRNA from nematodes incubated in siRNA of $M g$-pat-10 and $M g$-unc- 87 for $24 \mathrm{~h}$ showed that the transcript levels of the genes were reduced. This reduction was reflected in the nematodes activity. Part of the nematodes incubated in siRNA of $M g$-pat-10 was paralyzed while part of those incubated in $M g-u n c-87$ siRNA was coiled. Pat-10 is essential for the initial assembly of the sarcomere and is involved in the attachment of muscle cells to the basement membrane [22]. Although nematodes were paralyzed after incubation in siRNA of $M g$-pat-10, the pharyngeal pumping remained active. This agrees with the observation made by Terami et al. [23] who reported that pharyngeal pumping was unaffected in pat-10 mutants of the nematode C. elegans. Unc-87 serves as a structural component to maintain lattice integrity during contraction [24] and hence silencing of unc-87 leads to disorganized body wall muscles affecting the contraction/relaxation cycle of the muscles (uncoordinated movement). The migration assay that was performed on siRNA treated nematodes showed that silencing of $M g$-pat-10 and $M g-u n c-87$ significantly impedes nematode movement. These results demonstrate that the function of $u n c-87$ and pat-10 has been evolutionarily conserved between the plant parasitic nematode $M$. graminicola and the free living nematode C. elegans. Recently, it was also reported that silencing of unc-87 and pat-10 give a similar phenotype in Pratylenchus species [25,26].

Incubation of the nematodes with siRNA at the concentration of $50 \mathrm{ng} / \mu \mathrm{L}$ for $24 \mathrm{~h}$ appeared to induce gene knock down in M. graminicola. However, recovery in transcript level of $M g$-pat-10 and $M g-u n c-87$ was observed later. The recovery of transcript levels of $M g-u n c-87$ was faster, but not as complete as that of $M g$-pat-10. The transcript level of $M g$-pat-10 was the same as the control at $72 \mathrm{~h}$ after washing, while the transcript level of $M g-u n c-87$ started to be less silenced earlier, but did not yet approach the level of the control after $72 \mathrm{~h}$. This recovery from the siRNA treatment is similar to the observation of Soumi et al. [25] in Pratylenchus coffeae with the only difference being that they used long double stranded RNA. They found that most of the nematodes that were incubated in dsRNA of Pc-pat-10 recovered normal sinusoidal movement after $24 \mathrm{~h}$. On the contrary, the worms incubated in Pc-unc-87 showed the aberrant phenotype after $24 \mathrm{~h}$ and very few worms could regain regular movement. This time-limited silencing effect was also reported by Rosso et al. [27] after in vitro soaking of $M$. incognita in dsRNA of Mi-crt (calreticulin) and Mi-pg-1 (polygalacturonase) genes. While the silencing effect on Mi-crt lasted for $44 \mathrm{~h}$, the effect on Mi-pg-1 remained only for $20 \mathrm{~h}$ after soaking.

Dalzell et al. [20] revealed that on strategically incorporating base mismatches in the sense strand of a G. pallida specific siRNA, they could specifically increase or decrease the knockdown of its target (specific to the antisense strand). In our investigation, the siRNA with a mismatch did not produce any observable aberrant phenotypes and no difference was noticed in gene silencing between siRNAs with mismatch and the control treated nematodes.

Dalzell et al. [28] found that non nematode derived dsRNAs induced aberrant phenotypes and had an unexpected inhibitory effect on the motility of root-knot nematode $M$. incognita J2s following $24 \mathrm{~h}$ soaking in $0.1 \mathrm{mg} / \mathrm{mL}$ dsRNA, and established that this inhibitory phenomenon was both time- and 
concentration-dependent. In our experiments, we included as a control the silencing of a $\beta-1,4$-endoglucanase under the same conditions and it could be demonstrated that the siRNA of this gene $\mathrm{Mg}$-eng did not cause any inhibition of motility.

\section{Conclusions}

In conclusion, we have demonstrated that $M$. graminicola is susceptible to effective specific gene silencing by siRNAs. However, the persistence of the RNAi effect in M. graminicola is highly time limited and variable among the targeted genes. Therefore, it is essential to have a greater understanding of the RNAi regulatory pathway in plant parasitic nematodes to enhance the potency and persistence of RNAi.

This successful application of RNAi in M. graminicola could open the door for the identification of novel target genes that are essential to nematode biology and parasitism in order to control these root-knot nematodes in agriculture.

\section{Acknowledgements}

This research was funded by a doctoral grant of the Belgian Technical Cooperation (BTC-CTB) to Joseph Nsengimana and a postdoctoral research grant from the Flanders Research Foundation (FWO) to Annelies Haegeman.

The work was performed in the Department of Molecular Biotechnology in the Faculty of Bioengineering at Ghent University. The authors would like to thank Dirk De Waele from the Catholic University of Leuven for providing the starting culture of M. graminicola.

\section{Conflicts of Interest}

The authors declare no conflict of interest.

\section{References}

1. Nicol, J.M.; Turner, S.J.; Coyne, D.L.; den Nijs, L.; Hockland, S.; Maafi, Z.T. Current nematode treaths to world agriculture. Genomics Mol. Genet. Plant Nematode Interact. 2011, doi:10.1007/978-94-007-0434-3_2.

2. Jaiswal, R.K.; Kumar, D.; Singh, K.P. Relationship between growth of rice seedlings and time of infection with Meloidogyne graminicola. Libyan Agric. Res. Center J. Int. 2012, 3, 13-17.

3. Ahmed, R.; Gowen, S. Studies on the infection of Meloidogyne spp. with isolates of Pasteuria penetrans. Nematol. Mediterr. 1991, 19, 229-233.

4. Arguel, M.J.; Jaouannet, M.; Magliano, M.; Abad, P.; Rosso, M.N. siRNAs trigger efficient silencing of a parasitism gene in plant parasitic root-knot nematodes. Genes 2012, 3, 391-408.

5. Elbashir, S.M.; Harborth, J.; Lendeckel, W.; Yalcin, A.; Weber, K.; Tuschl, T. Duplexes of 21-nucleotide RNAs mediate RNA interference in cultured mammalian cells. Nature 2001, 411, 494-498. 
6. Fire, A.; Xu, S.; Montgomery, M.K.; Kostas, S.A.; Driver, S.E.; Mello, C.C. Potent and specific genetic interference by double-stranded RNA in Caenorhabditis elegans. Nature 1998, 391, 806-811.

7. Rosso, M.N.; Jones, J.T.; Abad, P. RNAi and functional genomics in plant parasitic nematodes. Annu. Rev. Phytopathol. 2009, 47, 207-232.

8. Kimber, J.K.; McKinney, S.; McMaster, S.; Day, A.T.; Fleming, C.C.; Maule, G.A. Flp gene disruption in parasitic nematode reveals motor dysfunction and unusual neuronal sensitivity to RNA interference. FASEB J. 2007, 21, 1233-1243.

9. Meissner, B.; Warner, A.; Wong, K.; Dube, N.; Lorch, A.; McKay, S.J.; Khattra, J.; Rogalski, T.; Somasiri, A.; Chaudhry, I.; et al. An integrated strategy to study muscle development and myofilament structure in Caenorhabditis elegans. PLoS Genet. 2009, 5 , e1000537.

10. Kranewitter, W.J.; Ylann, E.J.; Gimona, M. UNC-87 is an actin-bundling protein. J. Biol. Chem. 2001, 276, 6306-6312.

11. Yamashiro, S.; Gimona, M.; Ono, S. UNC-87, a calponin-related protein in C. elegans, antagonizes ADF/cofilin-mediated actin filament dynamics. J. Cell Sci. 2007, 120, 3022-3033.

12. Simmer, F.; Moorman, C.; van der Linden, A.M.; Kuijk, E.; van den Berghe, P.V.E.; Kamath, R.S.; Fraser, A.G.; Ahringer, J.; Plasterk, R.H.A. Genome-wide RNAi of C. elegans using the hypersensitive rrf-3 strain reveals novel gene functions. PLoS Biol. 2003, 1, e12.

13. Haegeman, A.; Bauters, L.; Kyndt, T.; Rahman, M.; Gheysen, G. Identification of candidate effector genes in the transcriptome of the rice root knot nematode Meloidogyne graminicola. Mol. Plant Pathol. 2013, 14, 379-390.

14. Translate a DNA Sequence. Available online: http://www.vivo.colostate.edu/molkit/translate/ (accessed on 10 July 2010).

15. Life Technologies Website. Available online: http://www.ambion.com/techlib/misc/siRNA_tools. html (accessed on 21 May 2010).

16. Biolegio Home Page. Available online: www.biolegio.com (accessed on 2 June 2010).

17. De Ahmed, F.; Raghava, G.P.S. Designing of highly effective complementary and mismatch siRNAs for silencing a gene. PLoS One 2011, 6, e23443.

18. Hooper, D.J.; Hallmann, J.; Subbotin, S. Methods for Extraction, Processing and Detection of Plant and Soil Nematodes. In Plant Parasitic Nematodes in Subtropical and Tropical Agriculture; Luc, M., Sikora, R.A., Bridge, J., Eds.; CABI: Wallingford, UK, 2005; pp. 53-86.

19. WormBase Website. Available online: www.wormbase.org/db/searches/blast-blast (accessed on 24 March 2010).

20. Dalzell, J.J.; McMaster, S.; Fleming, C.C.; Maule, G.A. Short interfering RNA-mediated gene silencing in Globodera pallida and Meloidogyne incognita infective stage juveniles. Int. J. Parasitol. 2010, 40, 91-100.

21. Rosso, M.N.; Favery, B.; Piotte, C.; Arthaud, L.; de Boer, J.M.; Hussey, R.S.; Bakker, J.; Baum, T.J.; Abad, P. Isolation of a cDNA encoding a $\beta$-1,4-endoglucanase in the root-knot nematode Meloidogyne incognita and expression analysis during plant parasitism. MPMI 1999, $12,585-591$. 
22. Williams, B.D.; Waterston, R.H. Genes critical for muscle development and function in Caenorhabditis elegans identified through lethal mutations. J. Cell Biol. 1994, 124, 475-490.

23. Terami, H.; Williams, B.D.; Kitamura, S.; Sakube, Y.; Matsumoto, S.; Doi, S.; Obinata, T.; Kagawa, H. Genomic organization, expression, and analysis of the troponin $\mathrm{C}$ gene pat-10 of Caenorhabditis elegans. J. Cell Biol. 1999, 146, 193-202.

24. Goetinck, S.; Waterston, R.H. The Caenorhabditis elegans UNC-87 protein is essential for maintenance, but not assembly, of body wall muscle. J. Cell Biol. 1994, 127, 71-78.

25. Joseph, S.; Gheysen, G.; Subramaniam, K. RNA interference in Pratylenchus coffeae: Knock down of Pc-pat-10 and Pc-unc-87 impedes migration. Mol. Biochem. Parasitol. 2012, 186, 51-59.

26. Tan, J.C.H.; Jones, M.G.K.; Fuso-Nyarko, J. Gene silencing in root lesion nematodes (Pratylenchus. spp.) significantly reduces reproduction in a plant host. Exp. Parasitol. 2013, 133, 166-178.

27. Rosso, M.N.; Dubrana, M.P.; Cimbolini, N.; Jaubert, S.; Abad, P. Application of RNA interference to root-knot nematode genes encoding esophageal gland proteins. Mol. Plant Microbe Interact. 2005, 18, 615-620.

28. Dalzell, J.J.; Warnock, N.D.; Stevenson, M.A.; Mousley, A.; Fleming, C.C.; Maule, G.A. Short interfering RNA-mediated knockdown of drosha and pasha in undifferentiated Meloidogyne incognita eggs leads to irregular growth and embryonic lethality. Inter. J. Parasitol. 2010, 40, 1303-1310.

\section{Appendix}

ESTs used for target genes and control.

A1. $>$ Mg_pat-10

GAATCAACGTTTTCCTCTCTTAATTTTTCTTTAAATCCCTAAAATTATTTAATTTTTAAAAT ATTTATTTTCAGTACCTCTTTTCTATTTTATACTCATAAATATTTGAAATGGCCGAAAATAT TGAAGAAATCCTTGCTGAAATTGACGGCTCTCAAATAGAGGAATATCAACGATTTTTTGA TATGTTTGACCGTGGAAAGAATGGTTACATTATGGCTACTCAAATTGGAACAATTATGAA TGCTATGGAACAAGATTTTGATGAGAAAACCCTTCGAAAATTAATCCGCAAATTCGACGC AGACGGCAGTGGTAAAATCGAATTTGATGAGTTTTGTGCATTAGTATACACTGTAGCAAA TACTGTAGATAAGGATACATTACGGAAAGAATTGAGAGAAGCTTTTCGTCTTTTCGACAA GGAGGGTAATGGTTACATCTCCCGCCCTACTCTTAAAGGACTTCTTCACGAAATTGCACC AGACCTAAGTGATAAAGATTTGGATGCTGCAGTAGATGAAATTGACGAAGACGGAAGCG GTAAAATTGAATTTGAAGAATTTTGGGAATTAATGGCTGGAGAGACTGATTAAAATTTAA AATAATTAAAATTAATATTTTGCCATAAAATAAAAAAGGTGCCCCTAAATTGCCTTTAAC AATTTTATTATTTT 
A2. $>$ Mg_unc-87

AGACCCTGCCGATTTGGAGCCTCTTCCAGAAGAAAAAATCCGTGCTAGTGATGGAATTGT TCGTTTACAATCCGGTACGAACAAGTTTGACTCACAAAAAGGAATGGTCAGCTTCGGTAC AAACCGACGCGAAACTACCAGAATGAAAGATACAAAACATCCGGAATATGATCACGAAC GCAATATTGATCAAAGCGAGATTCCTTTGCAATCCGGTACGAACAAATTTGCTTCACAAA AAGGAATGGTCAGCTTCGGTACAAACCGACGCGAAACTACAAAAATGTTGGATACTGCC CATCCGGAATACAGTCACGAAAGCAGTATTGACCAAACAAGTATTCCTTACCAAATGGGT TCAAATCGTTATGCTTCACAAAAGGGAATGACTTGTTTTGGACAACCACGTTGGGAGGTG CTTGACCCAAGTATTAGCTATCAGAATCGTAAATCACAAGGAATGGTTCGTCTTCAATCC GGTACAAACCGGTTTGCTTCACAAGCAGGAATGACAGGTTTTGGAACTCCAAGGAACACA ACATATGAAGCAGAAGCTGGAGAACTTCCTTATGAAGATATGAAAAAATCAGAAACAAT AATTCCATCCCAGGCTGGTTGGAATAAGGGAGACTCTCAAAAATTAATGACTGGATTTGG TACTCCACGTGACGTTAAAGGAAAACATTTGAAGCGTATATGGGAATTGGAATATCCAGA GGAGGCTGAAATTTCTTTAGATCGACTTTAAAGGAATTGAAAAGA

A3. $>$ Mg-eng

ATGTTTAAAAATTGCTTAATATTAAATATTTTATTTATTTCTTATTATTTATCTATTATATTA GCAGCTAACCCGCCTTATGGAAAATTATCAGTACGTAAAGGGCAGTTAAAGGGGTCGAAT GGCCAAGTAGCTACATTGAGAGGAATTTCTCTCTGGTTTAGTCAATGGATAACTGAATTTT ATTCGCCTGGTGTTGTAAGGGCAATAAAATGTTTTTATAATGGGAATGTTGTTCGTGCTTC AATTGGAACCTGTTGTTCAGGTTATTTGGAAAACCCATCTGCTGCAATAAATGCTGCCAC AACTATTGGAGATGCAGCAATTGCTAATGGAATGTATTTTATAATTGACTGGCATGATGT AGGAAATCAGAAATGTAATAATGACGCAGATTTTAACAACTTCATAAATAGTGCAATTAA ATTTTTTACAACAATTATTAACAAATATAAGGGCTCACCAAATATACTTTTAGAGCTTTGG AATGAACCTGCTTATACTTGTAGTTGGGCTAAATTGAAGCAATATTATAACGCTGTTCTTA CTCAAGTTAGAAAAATTGACCCAAATGTCGTCGTCATTCTCGGAACACCGAAACAGTCTA CTGGTCCAAGCGACGAAGTCATAAATAGTCCTGTTGGTGGTGCCAATATAATGTATGCCT TACATTTTTATTGCGTACCGTACCAACAACATATTGAAAATCAAAAACAAATGATATTAA AAGCAAAAAGTAAAGGATTTGCAACATTCGTTAGTGAATATGGCGATGCAGATGCAACC CCTCCAGCACCTCTTCAACCAACAGCAATGAAGGCATTTTGGCAATTTATGGACCAAAAC CAACTTTCCTATGCAAAATGGTCACTTACTAACAAAGATGAGGTTTATTCATTAATGGTGC CATGGTGTAGTGCATCGCAAGCATTTCAAGAATCATGTCTTAGTGCCTCAGGGAAATTGC TTAGAGAACATATGTGGAGCTTAAATAATGGTATTAATGGTTGTTAA

(C) 2013 by the authors; licensee MDPI, Basel, Switzerland. This article is an open access article distributed under the terms and conditions of the Creative Commons Attribution license (http://creativecommons.org/licenses/by/3.0/). 